It may be argued that the computed percentages of creosote, calculated on the basis of dry weight of the sawdust, are not the actual values which should be taken into consideration because of the amount of water present during the growth of the organisms. However, we feel that there would be little in favor of such an argument when compared with actual practice. In commercial practice the amount of impregnation is calculated as pounds of creosote per cubic foot of wood, seasoned either before or during the process of impregnation. Thus, the percentage of creosote ultimately becomes a question of weight, depending upon the specific gravity of the wood. Moreover, in actual practice, impregnated timbers, such as railroad ties, may be placed under moisture conditions very favorable to the support of fungous growth. Under these conditions, moisture would be absorbed by the wood. The present experiments were attempts to imitate these natural conditions which are conducive to timber decay.

\title{
Nitrocellulose and Its Solutions as Applied to the Manufacture of Artificial Leather ${ }^{1}$
}

By W. K. Tucker

Hercules Powder Co., 12 Forbes ST., Worcester, Massachusetts

Although nitrocellulose has been used in the manufacture of artificial leather for a good many years, very little has been printed which is of practical value to anyone wishing to make a study of the business. This has been due partly to the desire of those who have had practical experience to profit personally by their knowledge, and partly because many who have had the requisite experience have not been scientific men and have worked roughly by rule of thumb, being entirely unfamiliar with the underlying principles. The facts presented in this paper have long been known to scientific and practical men in the artificial leather business, but so far as I know have not been published, and are of interest to industrial chemists.

\section{Viscosity of Solutions}

The first important point to be considered in the use of nitrocellulose for this purpose is viscosity. In discussing the viscosity of nitrocotton it is always understood that reference is made to the viscosity of a 16-oz. solution of nitrocotton in some solvent. This standard solution is made by dissolving $16 \mathrm{oz}$. of nitrocellulose in a gallon of solvent. A certain increase in volume occurs during this process so that the standard 16-oz. solution contains somewhat less than 16 $o z$. of nitrocotton to the gallon of solution. For instance, in one of our standard solutions $16 \mathrm{oz}$. of nitrocotton added to 1 gal. of the mixture of solvents and non-solvents produced 1.965 gal. of solution, containing 12.2 per cent of nitrocotton. If the solution actually contained $16 \mathrm{oz}$. of nitrocellulose to the gallon the percentage would be 13 per cent.

The viscosity of nitrocellulose is determined by noting the time taken for a steel ball five-sixteenths in. in diameter to drop through a 10-in. column of a 16-oz. solution of the material in question. This would appear to be a simple method and one that could be used by any manufacturer. Unfortunately, a great deal of confusion occurs when customers order cotton specifying the viscosity by this method, because there is no standard solvent generally used for such a test, and varying temperatures and the size of the container affect the result. In this laboratory we use a standard 16-oz. solution (i.e., $16 \mathrm{oz}$. of cotton to the gallon of solvent), and the solvents are roughly 70 per cent ethyl acetate and 30 per cent benzene. A standard container, large enough in diameter to prevent any effects of friction from the displacement of the solution, is used, and the test is made at $25^{\circ} \mathrm{C}$.

In general, this is the method used by large consumers and manufacturers of nitrocellulose to determine viscosity, and it is accurate enough, providing the mixture of solvents and the apparatus be standardized so that conditions are identical when the test is made by the manufacturer and consumer. If this Socretr could get together the data

1 Presented before the Section of Cellulose Chemistry at the 61st Meeting of the American Chemical Society, Rochester, N. Y., April 26 to 29, 1921. required and standardize the method, a great service would be rendered to the industry, eliminating many unfortunate mistakes and misunderstandings.

It may be noted here that most of the cotton used for the manufacturing of artificial leather is of low viscosity. The problem is to fix the required amount of cotton and other solids on a cloth base with the least loss of solvents. As the viscosity of the final solution as applied is fixed within rather narrow limits by the coating machine, and as the solution must be as inexpensive as possible, it is obvious that a cotton is desirable which will allow of the addition of a large amount of cheap non-solvent, such as benzene, and yet not bring the viscosity of the solution above the set limits $(i . e$., about 40 sec.). As the addition of non-solvents always increases the viscosity of a solution, the low viscosity cotton is logically the grade most suitable, since it will admit of the addition of the most non-solvent.

Although the manufacture of nitrocellulose of various viscosities is too big a subject to enter into in this paper, the following generalities can be mentioned. A high viscosity cotton usually results from a high nitrogen content, or from a low temperature during nitration, while a high nitrating temperature or a long nitration results in a low viscosity product.

The industry uses nitrocotton of three viscosities: low, from 5 to 20 sec.; medium, from 40 to 60 sec.; and high, from 100 to $2400 \mathrm{sec}$. The greatest demand is for a material of about 20 sec. The higher viscosities are generally used for blending purposes to bring the viscosity of a certain solution up to the required point.

\section{Degree of Nitration}

Roughly stated, the problem is to place a certain amount of nitrocotton on the surface of the cloth with the least waste of solvents.

The second point to be considered is the conditions of nitration desirable in nitrocellulose to be used in the making of artificial leather. This is determined by the fact that varying degrees of nitration affect its solubility in the commonly used solvents.

The nitrogen content of such a material averages about 12 per cent, although a range of from about 11.5 to 13 per cent is allowable. Roughly speaking, the lower the nitrogen content between these limits, the greater the solubility, and consequently the greater the amount of non-solvents which can be used in solutions. If the nitrogen content goes above or below these limits, the solubility of the nitrocellulose in the usual solvents decreases; less non-solvents can be used, and finally particles of cotton fail to go into solution, remaining suspended in the solution.

\section{Stabilization}

The third point to consider is the question of stabilization 
and bleaching which are required after nitration. Except where cotton is to be used for making a white artificial leather, the question of color is not of the highest importance. Nevertheless, it is desirable to give the material a very thorough treatment in the boiling tubs, and produce a cotton which can be used on any grade of artificial leather. Opinion varies as to the importance of stability in nitrocellulose to be used for this purpose, but it is generally acknowledged that an unstable product will cause cracking of the film in the finished leatherette.

It is to be noted that the stabilization and pulping processes have a decided lowering effect on the viscosity of nitrocellulose, so that in making a cotton to meet a specified viscosity this element must be taken into account. Also this effect can be utilized to reduce the viscosity where it is found to be too high.

The test for ash is of minor importance where the nitrocellulose is to be used for the manufacture of artificial leather. Nevertheless, it is always made, as an excessive ash indicates the presence of too much dirt. A good grade of soluble cotton should not have over 0.4 per cent ash.

\section{SOLVENTS}

In making up solutions for the manufacture of artificial leather, two solvents are very generally used: acetone oils and ethyl acetate. The commercial acetone oils consist largely of methylethylketone, with some acetone and higher ketones, as well as small quantities of condensation products, such as mesityl oxide. The non-solvents are usually benzene or benzine. As nitrocellulose is usually shipped wet with denatured alcohol, this latter material is nearly always present in the final solution. Although not a perfect solvent for nitrocotton, alcohol tends to lower the viscosity of a solution. A typical artificial leather solution rarely contains more than 30 per cent acetone oils or other solvent, the remainder consisting of benzene, etc. This percentage is often reduced even to 10 per cent.

It used to be considered necessary to have present a certain amount of high boiling solvent, such as amyl acetate, ethyl propionate, etc., but most manufacturers now get along without its presence, and some claim that such a solvent actually tends to produce pinholes in the finished leather. This, they think, is caused by the higher boiling solvent remaining in the coat until most of the other solvents and non-solvents have evaporated. The non-use of high boiling solvent is, of course, made possible only by the use of a mixture in which the boiling point of the solvent is higher than that of the non-solvent, or where their boiling points are very nearly the same, as otherwise the rate of evaporation of the solvent during the drying process would be greater than that of the non-solvent. In such a case a point would be reached where the percentage of non-solvent would be high enough to precipitate part of the nitrocotton and cause blushing of the film and a consequent flaking off of the coat.

The solutions usually used by manufacturers have a viscosity of about 40 sec. It is interesting to note that the solvents and non-solvents used have a decided effect on the viscosity of a solution. In general, it may be stated that solvents of higher molecular weight make a more viscous solution when nitrocotton is dissolved in them than do those of lower molecular weight. Thus, solutions in ethyl propionate, ethyl butyrate, butyl acetate, or amyl acetate are of higher viscosity than solutions of the same nitrocellulose in acetone or ethyl acetate. The introduction of any non-solvent always increases the viscosity of the resultant solution. Thus, nitrocellulose which will give the standard 16-oz. solution a viscosity of $20 \mathrm{sec}$. when dissolved in a mixture of 70 per cent ethyl acetate and 30 per cent benzene will give a viscosity of about $40 \mathrm{sec}$. when the percentage of benzene is
70 and that of the ethyl acetate 30 .

Solutions in which the viscosity is too high are generally known as "short" solutions. "Granular" solutions are those which are hazy and in which all the nitrocellulose has not dissolved. These latter are usually caused by either over- or under-nitration of the cotton, making it partly insoluble in the usual solvents.

There exists a good deal of difference of opinion as to the relative advisability of using a 16-oz. solution or one where the nitrocotton content is higher, such as a 20 - or 24-oz. solution. The majority of manufacturers seem to use the 16-oz. solution, but there are notable exceptions. Men well up in the business declare that the cost of manufacture depends largely on cost of materials, the labor element being small. It would appear on the surface, then, that if an artificial leather of the same weight could be made by the application of fewer coats of a dope containing more nitrocotton, considerable economy might be effected in the saving of solvents. However, there are a good many factors to consider. A 24-oz. solution would have to be considerably richer in solvents to give a viscosity of 40 sec. than a 16-oz. solution giving the same viscosity. That is, the proportion of cheap non-solvent would have to be decreased, and the mixture would rise in cost per gallon. Reducing the viscosity of the cotton used would not be very advantageous, as the extremely low viscosity cotton is uneconomical to manufacture on account of the low yields and danger of fires. Some also claim that the heavier solutions tend to produce more pinholes in the finished goods. Probably there is room for difference of opinion on this point, and a practical man who has developed a good product using a certain weight solution is naturally averse to making a change. After the nitrocellulose and the solutions are brought up to a satisfactory standard, a great deal has yet to be done to turn out a high-grade artificial leather, and it is here that the actual practical operator has a field for valuable experimental work.

\section{Monthly Statistical Service on Production and Stocks of Sulfuric Acid and Nitric Acid}

The Department of Commerce, through the Bureau of the Census, is preparing to issue monthly reports on the production and stocks of sulfuric acid and nitric acid. Secretary Hoover has been assured of the coöperation of the manufacturers of these products, and Mr. Steuart, Director of the Census, is consulting them as to the form of schedule that will yield the required data with least inconvenience to the industry. The data secured from firms and corporations will be held as confidential, totals only being published.

The purpose of the Department in issuing monthly reports of this character is to assist in the stabilization of industry by furnishing such aid as may be possible by the dissemination of information necessary to intelligent procedure. The undertaking is endorsed by the Manufacturing Chemists' Association, and by many officials of corporations engaged in the production of these acids. This is an opportunity for the chemical industry to secure an important service from the Department of Commerce, and it is recommended that all manufacturers who receive the schedule give it prompt attention and assist in the compilation of reports that can be distributed shortly after the end of the month to which the figures relate.

\section{Insecticide and Disinfectant Manufacturers Association}

The recent meeting of the Insecticide and Disinfectant Manufacturers Association at Atlantic City, June 13 and 14, 1921, was a success from the point of view of attendance and interest shown. Among the guests who addressed the Association was Mr. W. D. Hartley, representative of McDougall Bros., Ltd., Manchester, England, who discussed informally trade conditions in England. 